\title{
IMPACTO DA EXPRESSÃO FACIAL NA PERCEPÇÃO DE TEMPO: PAPEL DA VALÊNCIA E DA ACTIVAÇÃO (AROUSAL)
}

\author{
Alexandre Fernandes 1 \\ Teresa Garcia-Marques ${ }^{2}$
}

Resumo: A natureza emocional de uma expressão facial parece enviesar a percepção da sua duração. Neste artigo apresentamos um estudo que testa a hipótese deste efeito ser moderado pelas dimensões emocionais (valência e activação [arousal]), abordando a sua explicação em termos de processos que interferem com o funcionamento de um "relógio interno", nomeadamente processos atencionais e activacionais. Utilizando uma tarefa de bissecção temporal, com durações entre 400 e $1600 \mathrm{~ms}$, os resultados sugerem um efeito da valência da emoção expressa por uma face: a duração das faces negativas é sobre-estimada relativamente às positivas. Este efeito foi moderado pela intensidade das expressões emocionais (activação [arousal]) verificando-se apenas claramente na condição de baixa intensidade. $\mathrm{Na}$ condição de elevada intensidade, as faces positivas foram avaliadas de forma idêntica às negativas $O$ facto de esta interacção apenas se verificar em durações mais curtas, sugere uma interferência concorrente de processos atencionais e activacionais. Esta explicação é discutida no artigo, tendo em consideração as suas limitações.

Palavras-chave: expressão facial, percepção de tempo, emoção, valência, activação (arousal)

The impact of facial expression on time perception: The role of valence and arousal (Abstract): The emotional nature of a facial expression seems to favour a bias regarding the perception of its duration. In this paper we present a study that tests the hypothesis that this effect will be moderated by the emotional dimensions of facial expressions (valence and arousal), addressing its explanation in terms of processes that interfere with the functioning of an "internal clock", including activation and attentional processes. Using a temporal bisection task with duration

\footnotetext{
${ }^{1}$ Instituto Superior de Psicologia Aplicada, Lisboa

2 Instituto Superior de Psicologia Aplicada, Lisboa

Correspondência: Alexandre Fernandes. UIPCDE - Unidade de Investigação em Psicologia Cognitiva, do Desenvolvimento e da Educação. ISPA - Instituto Universitário. Rua Jardim do Tabaco, 34, 1149-041 Lisboa; Tel.: 218811 700; E-mail: alexandre@ispa.pt ou gmarques@ispa.pt
}

PSICOLOGIA, Vol. XXIV (2), 2010, Edições Colibri, Lisboa, pp. 61-88 
between 400 and $1600 \mathrm{~ms}$, the results suggest a valence effect: The duration of the negative faces is overestimated in relation to positive faces. This effect was moderated by the intensity of emotional expressions (arousal), but only in the low arousal condition. In the high arousal condition positive and negative faces were identically estimated. The fact that this interaction has been found only in short time windows, suggests a concurrent interference of activation and attentional processes. This explanation is discussed in the article, taking into account its limitations.

Keywords: facial expression, time perception, emotion, valence, arousal

\section{Introdução}

A experiência de distorções temporais associadas a eventos emocionais é um fenómeno que todos nós já experimentámos. Quem não sentiu o "tempo voar" quando vivemos momentos muito agradáveis, ou que o tempo "não passa" quando estamos aborrecidos à espera numa fila interminável. Durante experiências traumáticas, é comum as pessoas relatarem que o tempo parece abrandar, e que os eventos duram mais tempo do que na realidade duram (Noyes \& Kletti, 1977; Ursano, Fullerton, Epstein, Crowley, Vance, \& Kao, 1999). De modo a caracterizar e compreender estes fenómenos do senso comum, vários investigadores procuraram replicar estas experiências subjectivas em laboratório e estudá-las de forma controlada. Apesar da natureza multifacetada destes fenómenos ${ }^{3}$, o estudo da influência das emoções na percepção de tempo tem sido restringido apenas à percepção de duração de eventos (ou estímulo) emocionais. É de referir que a percepção de tempo enquadra diferentes experiências elementares como a simultaneidade, a sucessividade, o julgamento de ordem temporal, a percepção de ritmo, a percepção de fluxo (passagem do tempo) e a percepção de intervalo de tempo ou duração (e.g., Block, 1990; Fraisse, 1984; Friedman, 1990; James, 1890); Pöppel, 1978). Esta noção tem sido acompanhada de evidências que estas experiências elementares de tempo estão associadas a diferentes mecanismos de processamento temporal (ver Fraisse, 1984). Este artigo visa con-

\footnotetext{
${ }^{3}$ Por exemplo, percebemos um evento constituído por variadas e interessantes experiências passando muito rápido, mas quando nos recordamos desse evento parece que durou mais tempo. Por outro lado, um evento vazio de experiências parece demorar uma eternidade, mas quando olhamos retrospectivamente parece-nos mais curto. Este paradoxo reflecte diferentes fenómenos, um relacionado com o fluxo (sucessão de unidades de tempo) e outro com a duração (número de unidades de tempo).
} 
tribuir para o entendimento dos mecanismos subjacentes à influência das emoções na percepção de duração de estímulos emocionais.

O que se sabe sobre a influência das emoções na percepção de duração de um evento? Há 120 anos atrás já William James (1890), seguido por outros autores (e.g., Fraisse, 1957; Surt, 1925), chamava a atenção para a influência que as emoções exercem na nossa percepção de tempo. É, hoje em dia, considerável o número de investigações que sugere que os afectos interferem na percepção de duração. Este efeito é verificado em diversos estudos laboratoriais que utilizaram como estímulos faces com várias expressões emocionais (Droit-Volet, Brunot, \& Niedenthal, 2004; Effron, Niedenthal, Gil, \& Droit-Volet, 2006; Fernandes \& Garcia-Marques, 2008b; Fernandes, Garcia-Marques, \& Sá, 2006; Gil, Niedenthal, \& Droit-volet, 2007; Mondillon, Niedenthal, Gil, \& Droit-Volet, 2007; Tipples, 2008; Xuan \& Xiaolin, 2007), sons agradáveis e desagradáveis (Fernandes \& Garcia-Marques, 2008a; Noulhiane, Mella, Samson, Ragot, \& Pouthas, 2007), imagens positivas e negativas de cenários reais (Angrilli, Cherubini, Pavese \& Manfredini, 1997) e cheiros agradáveis e desagradáveis (Schiff \& Thayer, 1968). Do mesmo modo, verificaram-se interferências na percepção de duração em estudos que manipularam o estado afectivo dos indivíduos por apresentação de filmes (Chebat \& Gelinas-Chebat, 1995), por instruções que suscitavam a antecipação de choques eléctricos (Langer, Wapner, \& Werner, 1961), por promoção de contacto real com animais activadores de fobias (Watts \& Sharrock, 1984), por procedimento de Velten (Hawkings, French, Crwford, \& Enzle, 1988), por promoção de interacção com pessoas zangadas ou alegres (Thayer \& Schiff,1975), por indução de dor (Orme, 1969), ou por criação de expectativas positivas ou negativas (Edmonds, Cahoon, \& Bridges, 1981; Schiff \& Thayer, 1970). O enviesamento na percepção de duração é igualmente verificado por manipulação de estados afectivos em situações "naturais" (fora do laboratório), como situações de saltos-em-queda de plataforma ("bungie jumping"; Stetson, Fiesta, \& Eagleman, 2007) ou saltos de pára-quedas ("skydiving"; Campbell \& Bryant, 2007).

$\mathrm{O}$ que nos dizem estes estudos? Atendendo às pesquisas que permitem uma comparação de condições "afectivas" com condições de controlo não-afectivas (neutras) ${ }^{4}$, diremos que as estimativas de duração dos estímulos emocionais são sobrestimadas face às neutras (Angrilli et al., 1997; Droit-Volet et al., 2004; Effron et al., 2006; Fernandes et al., 2006; Fernandes \&

\footnotetext{
${ }^{4}$ Quando utilizamos um referencial absoluto, os enviesamentos são moderados pelo tipo de tarefa de estimativa temporal (Block, \& Zakay, 1997; Gupta, \& Cummings, 1986), impossibilitando a comparação directa entre estudos. Por exemplo, as estimativas de duração recorrendo a escalas temporais traduzem-se sistematicamente em sub-estimativas face ao tempo físico real.
} 
Garcia-Marques, 2008b; Gil et al., 2007; Langer et al., 1961; Mondillon et al., 2007; Noulhiane et al., 2007; Schiff \& Thayer, 1968; Schiff \& Thayer, 1970; Stetson et al., 2007; Tipples, 2008; Watts \& Sharrock, 1984). Tal é verdade, independentemente de se tratar de um afecto induzido no participante (e.g., Langer et al., 1961), ou de um afecto associado ao estímulo a ser avaliado na sua duração (e.g., Gil et al., 2007)5. O mesmo efeito parece ser independente do tipo de tarefa, de estimativa de duração ${ }^{6}$. Quer se trate de uma tarefa de bissecção, reprodução, ou estimativa verbal ou mesmo de uma escala temporal (milissegundos a minutos), as condições de natureza afectiva são sobre-estimadas relativamente às neutrais. São excepções os estudos onde se verifica uma sub-estimativa (Xuan \& Xiaolin, 2007), ou onde não é detectada diferença nas estimativas de duração entre os estímulos neutros e os emocionais (Hawkings et al., 1988; Thayer \& Schiff, 1975).

Que explicações têm sido avançadas para o facto de condições afectivas promoverem sobre-estimação? Este efeito tem, classicamente, sido enquadrado por diferentes modelos que têm em comum o facto de postularem a existência de um 'relógio interno' (e.g., Block, 1990; Gibbon \& Church, 1984; Gibbon, Church, \& Meck, 1984; Treisman, 1963). Estes $\operatorname{modelos}^{7}$, pressupõem este 'relógio' como um processador temporal constituído por um 'pacemaker' que emite pulsos a uma determinada frequência, um interruptor ('switch'), que é accionado pelos estímulos externos e que, com latências específicas, controla o acesso a um acumulador que soma os pulsos até ao término do evento. Deste modo, o número de pulsos registados

\footnotetext{
${ }^{5}$ Existe uma vasta literatura que demonstra que a própria natureza emocional dos estímulos promove reacções emocionais condizentes no percipiente (ver Niedenthal, 2007), apesar de, em rigor, não ser possível demonstrar que são os mesmos processos envolvidos (na indução emocional e na percepção de estímulos emocionais), e por isso afectarem o processamento temporal da mesma forma, mesmo que o efeito se observe na mesma direcção.

${ }^{6}$ As tarefas temporais mais utilizadas em paradigmas prospectivos têm sido as tarefas de bissecção temporal, reprodução temporal e a utilização de escalas de pontos ou analógicas. A tarefa de bissecção consiste na estimativa da duração de estímulos por comparação com as durações extremas (curta e longa) de uma série temporal, numa fase anterior de treino. Por outro lado, a reprodução temporal cifra-se na reprodução da duração de estímulos apresentados através da marcação do início e do fim do intervalo temporal estimado como igual à duração de apresentação do estímulo. As tarefas que recorrem a escalas intervalares ou contínuas consistem em procedimentos intermédios de classificação e reprodução, por cotação da duração dos estímulos.

${ }^{7}$ Os modelos de 'relógio interno' são modelos de processamento de informação, compostos por múltiplos componentes. Mas apesar de não encontrarem um suporte neurofisiológico robusto, continuam a ser o conjunto de modelos que faz predições relativamente precisas sobre a interferência de outros processos não temporais na percepção de tempo, ao contrário dos próprios modelos neurais (e.g., Buhusi \& Meck, 2006) e outros (e.g., comportamentais, BET, Killeen \& Fetterman1988; decaimento mnésico, Stadon \& Higa, 1999); não obstante o conceito de 'ticks' (pulsos) deste relógio continuar a ser hipotético.
} 
no acumulador constituirá a representação da duração percebida. Os enviesamentos temporais surgem em função de interferências em algum dos vários componentes deste relógio (Gibbon \& Church, 1984).

Como interferem os afectos nestes componentes? Segundo os autores dos estudos acima referenciados, é a dimensão de activação (arousal), associada à experiência emocional (sempre mais elevada do que aquela e associada a experiências neutras), a que mais interfere com o funcionamento do relógio. Esta dimensão dos afectos tende a qualificar a intensidade de uma experiência afectiva (que varia entre um estado muito calmo e/ou adormecido e um estado muito excitado e energético ${ }^{8}$. Pressupõe-se que esta intensidade afectiva incrementa a velocidade do relógio interno. Como consequência, verificamos um maior número de pulsos acumulados na memória de trabalho, que se traduzem directamente numa sobre-estimativa da duração dos estímulos (ver Droit-Volet \& Meck, 2007, para uma revisão). Para testar esta hipótese explicativa, alguns estudos procuraram manipular isoladamente os níveis de activação através: a) da administração de substâncias psicoestimulantes que têm impacto no sistema dopaminérgico (e.g., cocaína, Cheng, MacDonald, \& Meck, 2006; metanfetaminas, Maricq, Roberts, \& Church, 1981; para uma revisão ver Buhusi, 2003), b) da manipulação da temperatura corporal associada à regulação homeostática e dos ritmos cronobiológicos (e.g., Fox, Bradbury, Hampton, \& Legg, 1967; Wearden \& Penton-Voak, 1995), c) da actividade física (e.g., Vercruyssen, Hancock, \& Mihaly, 1989) ou d) da estimulação sensorial (e.g., ruído ambiental, Delay \& Mathey, 1985, alta frequência de apresentação de estímulos visuais, Droit-Volet \& Wearden, 2002; Penton-voak, Edwards, Percival \& Wearden, 1996; Treisman, Cook, Naish, \& McCrone, 1994). Todos estes estudos sugerem que um aumento de activação fisiológica resulta em sobre-estimativas temporais.

Este hipótese sugere um papel relevante da experiência fisiológica associada aos afectos, o que é corroborado, por exemplo, pelo trabalho de Effron e colaboradores (2006). Estes autores mostraram que o efeito de sobre-estimativa da duração de faces expressando raiva é eliminado quando se impede ao percipiente a mímica da expressão facial ${ }^{9}$ (mantendo um lápis

\footnotetext{
${ }^{8}$ De um ponto de vista psicofisiológico, a activação emocional é um constructo que envolve múltiplos componentes interligados de um mesmo fenómeno; tendo uma componente subjectiva da intensidade afectiva percebida, uma contraparte expressiva (e.g., expressão facial, comportamento motor) e outra de activação fisiológica (e.g., frequência cardíaca, tensão muscular, actividade neuronal). Não existem evidências de qual destas componentes é mais relevante para o efeito de sobre-estimativa temporal da activação.

${ }^{9}$ Embora a expressão de uma determinada emoção (como a raiva) possa induzir diferentes emoções no observador (como medo, Atikson \& Adolphs, 2005), explicando o efeito de activação, os processos de simulação motora ('embodiment') parecem igualmente contribuir para a experiência emocional equivalente à expressão facial observada (ver Nieden-
} 
entre os lábios). A inibição da actividade muscular facial (associada a activação fisiológica) parece impedir a aceleração do relógio interno e a subsequente sobre-estimativa temporal.

A hipótese explicativa com base na activação sugere-nos uma variação linear na estimativa da duração dos estímulos, à medida que a experiência emocional se torna mais intensa. Na realidade, tanto Fernandes e Garcia-Marques (2008a), que apresentaram sons agradáveis de vários níveis pré-testados de activação, como Langer et al. (1961), que induziram expectativas de perigo crescente nos seus participantes, apresentaram dados que revelam uma sobre-estimativa temporal em função do aumento do nível de activação. No entanto, Noulhiane et al. (2007), que também manipularam a activação utilizando sons, encontraram um efeito de sub-estimativa para os estímulos mais emocionais. Também Angrilli et al. (1997), com a apresentação de imagens que suscitam vários níveis de activação fisiológica não encontraram qualquer efeito geral da manipulação. Parece ser, assim, possível que outros factores associados à percepção e à experiência emocional estejam a moderar o efeito da activação nas estimativas temporais.

$\mathrm{O}$ moderador mais imediato é aquele que se associa à própria experiência afectiva: a valência dessa experiência. Na realidade, duas dimensões ou factores (contínuos) têm sido sugeridos para caracterizar a (maior) variabilidade da experiência (sentimento) e expressão emocional ${ }^{10}$, a sua activação e a sua valência (ver Bradley, 2000). Tal como sugere o modelo circumplexo de Russell (1980), é em torno desta estrutura bidimensional ortogonal, onde a activação varia de 'pouco intenso' a 'muito intenso' e a valência varia de 'muito positiva, ou agradável' a 'muito negativa ou desagradável', que se estruturam todas as emoções. Pode, então o valência ser o moderador do efeito de activação? Do conjunto de estudos que testaram o impacto da valência afectiva na percepção de tempo, não emerge qualquer padrão consistente. Por exemplo, Edmonds et al. (1981), através da indução de expectativas afectivas, Hawkings et al. (1988), que manipulam o estado de espírito e Xuan e Xiaolin (2007), que utilizam faces emocionais, observaram uma sobre-estimativa temporal dos eventos positivos comparativamente à dos negativos. Ao utilizarem faces de diferentes expressões emocionais, os estu-

thal, 2007). Várias linhas de evidência sugerem que os indivíduos imitam automaticamente as expressões faciais percebidas produzindo alterações fisiológicas correlacionadas com os estados emocionais (Levenson et al., 1990).

10 Para além desta abordagem dimensional existe outra mais funcionalista, mas complementar, que caracteriza as emoções em estados discretos ou em categorias. Os modelos categoriais destacam a existência de um número finito de emoções básicas com características específicas, que de um ponto de vista funcional não se podem reduzir a um conjunto reduzido de dimensões (e.g. Ekman, 1992; Plutchick, 1962). 
dos de Droit-Volet et al. (2004), Effron et al. (2006), Thayer e Schiff (1975) e Tipples (2008) encontraram um padrão inverso. Por outro lado, os resultados de Fernandes et al. (2006), utilizando também faces emocionais, não revelaram quaisquer efeitos gerais da valência na percepção temporal. Porém, Angrilli e colaboradores (1997), fornecem-nos uma resposta directa à nossa questão, ao contrastarem num mesmo estudos os efeitos da valência e da activação. Tal permite perceber se os efeitos de activação são ou não moderados pela valência. Angrilli e colaboradores (1997) utilizaram imagens da 'International Affective Picture System' (IAPS, Lang et al., 1995), que se dissociavam em termos de valência e activação. Os seus resultados sugerem que estes dois factores interagem. Na condição de elevada activação, a duração das imagens percebidas como desagradáveis (e.g., bebé com tumor ocular, cabeça esmagada) foi sobre-estimada relativamente às imagens percebidas como agradáveis (e.g., cenas eróticas). Na condição de baixa activação, as imagens desagradáveis (e.g., animais mortos, rato no lixo) foram sub-estimadas comparativamente às imagens agradáveis (e.g., animais de estimação, bebé sorridente).

Porque pode a valência interferir com a forma como a activação influencia a percepção de duração? Segundo Angrilli e colaboradores (1997), tal pode acontecer porque os estímulos emocionais, em especial os negativos, captam a nossa atenção, e tal facto interfere, como se sabe, com o funcionamento do relógio interno. Segundo os modelos de relógio interno que integram um interruptor('switch') que inicia a contagem dos pulsos (Block, 1990; Gibbon \& Church, 1984; Gibbon, Church, \& Meck, 1984; Treisman, 1963), a duração subjectiva está directamente relacionada com os recursos atencionais dedicados ao processamento temporal. Em paradigmas de estimação temporal prospectivos, em que o indivíduo sabe à partida que vai estimar a duração de um estímulo/evento, a atenção é dedicada primordialmente à informação temporal desse estímulo. ${ }^{11}$ Se esta é desviada por elementos distractores (e.g., tarefa secundária, expectativa), ou os recursos atencionais são repartidos com outros processos desencadeados de modo automático (e.g., configuração perceptiva, novidade do estímulo), a duração subjectiva é tipicamente sub-estimada face ao tempo físico real (e.g., Brown, 1997; Casini \& Macar, 1997; Macar, Grondin, \& Casini, 1994; Zakay \& Yehoshua, 1989). Portanto, activação e atenção apresentam efeitos concorrentes (opostos) no relógio interno, e consequentemente nas estimativas temporais. Dado que os eventos negativos têm tendência para se destacar dos

11 Todos os estudos citados neste artigo utilizaram uma abordagem prospectiva para investigar as influências das emoções na percepção de tempo. Este paradigma prospectivo diferencia-se do retrospectivo em que o indivíduo sabe que vai fazer o julgamento temporal depois do evento, ou seja, não atende explicitamente às suas características temporais. 
positivos (e.g., Fox, Lester, Russo, Bowles, Pichler, Dutton, 2000; Öhman, Lundqvist, \& Esteves, 2001), Angrilli et al. (1997) concluíram que um mecanismo atencional (i.e., resposta de orientação, e.g., Sokolov, 1963) é preponderante na percepção temporal de eventos de baixo nível de activação, sendo as diferenças de valência aqui mais notadas. Neste caso, como mais recursos atencionais são desviados para as características perceptivas dos estímulos negativos, menos recursos são dedicados ao processamento do tempo, traduzindo-se numa sub-estimativa temporal. Este efeito da atenção em elevado nível de activação seria minimizado, porque os estímulos negativos desencadeariam uma resposta defensiva, com o consequente aumento da actividade do sistema nervoso autónomo (e.g., aumento da pressão sanguínea, tensão muscular, dilatação pupilar, ver Bradley, 2000; Cuthbert, Bradley, \& Lang, 1996) que estaria relacionado com a aceleração do relógio interno.

Se esta explicação se adequa ao padrão de interacção encontrado por Angrilli e colaboradores (1997), ela é desafiada pelos dados obtidos subsequentemente por Noulhiane et al. (2007). Estes autores utilizaram as mesmas tarefas temporais (i.e., reprodução e escala temporal) que Angrilli et al. (1997), mas um conjunto de estímulos de natureza diferente: sons (i.e. 'International Affective Digital Sounds System', IADS, Bradley \& Lang, 1999). Os seus dados reflectem apenas dois efeitos principais, e não a interacção previamente discutida. Incongruentemente com a hipótese atencional, os sons negativos foram julgados como de maior duração que os positivos. E de forma incongruente com os dados gerais, os estímulos de baixa activação foram percebidos como tendo maior duração. Interessante é que os autores interpretam estes dados com base numa interferência entre activação e valência. Pressupõe-se que os estímulos negativos induzem um aumento na activação (promovendo uma maior sobre-estimativa temporal). Por outro lado, pressupõe-se que os estímulos de elevada activação captem mais atenção, diminuindo os recursos atencionais dedicados à informação temporal e consequentemente promovendo uma sub-estimativa temporal.

Em conjunto, os estudos de Angrilli e colaboradores (1997) e de Noulhiane e colaboradores (2007) apresentam padrões de dados (e interpretações) aparentemente incongruentes, criando a necessidade de clarificação dos mesmos. Para o efeito, no presente estudo utilizaram-se como estímulos faces emocionais: um tipo de estímulos para o qual somos peritos no processamento, e que mantém constante o seu grau de complexidade e a sua distribuição temporal e espacial12, permitindo variar as emoções associadas com

12 Vários estudos têm demonstrado que a complexidade dos estímulos tem impacto na percepção temporal (e.g., Block, 1992). A dispersão e distribuição espacial e temporal parecem igualmente interferir com os mecanismos de processamento temporal (e.g., Penton- 
simples modificações na face. Na realidade, um conjunto de pistas perceptivas mínimas (e.g. um simples levantar dos cantos da boca) confere-lhe o seu cariz emocional. Embora este tipo de estímulos tenha características particulares ${ }^{13}$, o facto de existirem vários estudos de percepção temporal que utilizaram faces (embora só controlando a valência) (Droit-Volet et al., 2004; Effron et al., 2006; Fernandes et al., 2006; Fernandes \& Garcia-Marques, 2008a; Gil et al., 2007; Mondillon et al., 2007; Thayer \& Schiff, 1975; Tipples, 2008; Xuan \& Xiaolin, 2007) permite-nos interpretar os efeitos de uma forma comparativa. Tal não sucede com os estudos de Angrilli e colaboradores (1997) e Noulhiane e colaboradores (2007).

O objectivo do presente estudo foi abordar dois mecanismos (activação e atenção) passíveis de explicar os efeitos das emoções na percepção de duração. Utilizámos uma tarefa de bissecção temporal ${ }^{14}$ (similarmente aos estudos de Droit-Volet et al., 2004, Effron et al., 2006; Fernandes et al., 2006; Fernandes \& Garcia-Marques, 2008a; Gil et al., 2007; Mondillon et al., 2007) que consiste numa primeira fase de treino em que os participantes aprendem a distinguir uma duração curta de uma duração longa; e numa segunda fase de teste, em que classificam um conjunto de durações intermédias com base nas suas semelhanças com as durações extremas de referência (curta e longa). Os estímulos críticos apresentados na fase de teste foram faces expressando diferentes emoções: neutra (referência), positiva (alegria), e negativa (raiva). As faces emocionais foram apresentadas com dois níveis de expressividade (pouco intensa vs. muito intensa).

Considerando o efeito descrito do impacto dos afectos na percepção de duração (Angrilli et al., 1997; Droit-Volet et al., 2004; Effron et al., 2006; Fernandes et al., 2006; Fernandes \& Garcia-Marques, 2008b; Gil et al., 2007; Langer et al., 1961; Mondillon et al., 2007; Noulhiane et al., 2007; Schiff \& Thayer, 1968; Schiff \& Thayer, 1970; Stetson et al., 2007;

-voak et al., 1996). Tem sido demonstrado que, por exemplo, os estímulos sonoros, ao variarem temporalmente, permitem extrair pistas temporais que os estímulos estáticos não permitem (van Wassenhove, Buonomano, Shimojo \& Shams, 2008).

13 As faces humanas, apesar de serem estímulos visuais, apresentam características particulares do ponto de vista de processamento. São o veículo essencial da expressão emocional e de particular importância na sinalização e comunicação dos estados afectivos interindividuais (Bradley, 2000). Apresentam um processamento holístico (Tanaka \& Farah, 2003), rápido (Öhman, \& Soares, 1994), de destaque relativamente a outros estímulos (Öhman, Lundqvist, \& Esteves, 2001) e áreas cerebrais específicas para esse processamento (córtex infero-temporal, Sabatinelli, Bradley, Fitzsimmons, Lang, 2005).

14 A tarefa de bissecção, ao utilizar uma série com vários pontos temporais (neste caso sete: $400,600,800,1000,1200,1400,1600 \mathrm{~ms}$ ) permite diminuir a variabilidade associada a estimativas referentes apenas a pontos absolutos (i.e., durações específicas: por exemplo, 2 s; Angrilli et al. 1997). Por outro lado, permite igualmente extrair outros parâmetros psicofísicos (e.g., ponto de bissecção, integrais da função). 
Tipples, 2008; Watts \& Sharrock, 1984) esperamos que a percepção de duração dos estímulos emocionais seja sobre-estimada relativamente à duração dos estímulos neutros.

Esperamos igualmente replicar estudos anteriores que demonstram que as faces expressando raiva são percebidas durando mais tempo do que as faces expressando alegria (Droit-Volet et al., 2004; Effron et al., 2006; Thayer \& Schiff, 1975; Tipples, 2008). O facto de os estímulos negativos (neste caso, faces de raiva) tenderem a associar-se a níveis mais elevados de activação (e.g., Cacioppo \& Gardner, 1999; Russel, 1983) sustenta esta previsão. No entanto, este efeito deverá ser moderado pela intensidade das expressões emocionais e pela própria duração dos estímulos, em função de diferentes mecanismos. Por um lado, sabemos que os eventos negativos, neste caso as faces de raiva, captam mais a atenção (e.g., Fox et al., 2000; Öhman, Lundqvist, \& Esteves, 2001), e os indivíduos apresentam maiores latências a desfocar a atenção dessas expressões (e.g., Bonifacci, Ricciardelli, Lugli, \& Pellicano, 2008; Fox, Russo, \& Dutton, 2002;), e por isso competem com os recursos atencionais dedicados à percepção da informação temporal. Por outro lado, o nível de activação induzido na percepção das faces emocionais tem características dinâmicas, isto é, varia ao longo do tempo, aumentando numa primeira fase (em que o estimulo é apresentado) e decrescendo posteriormente, (e.g., Fridja, Ortony, Sonnemans, \& Clore, 1992). Evidências sobre a variação da activação fisiológica em função da duração do estímulo (e.g., Winkielman \& Cacioppo, 2001; Almeida, van Asselen, \& Castelo-Branco, 2010) corroboram também esta observação. Ora, as análises psicofísicas com base nos modelos de relógio interno têm sugerido que a activação parece ter um efeito multiplicativo, acelerando cada vez mais o relógio à medida que o tempo passa ${ }^{15}$, enquanto a atenção parece ter um efeito temporal aditivo, isto é, o efeito é uma constante independente da verdadeira duração do estímulo (ver Burle \& Casini, 2001; Droit-Volet \& Wearden, 2002). Neste sentido, quando estamos na presença de efeitos concorrentes da activação e da atenção, é de esperar que os efeitos atencionais se manifestem em durações mais breves. Ou seja, a perda de unidades temporais devido ao foco de atenção no estímulo sobrepor-se-á ao efeito de aceleração do relógio interno (mais unidades de tempo) promovido pela activação. Em durações mais longas o efeito da activação deve, então, sobrepor-se ao da atenção,

15 A abertura e fecho do interruptor ('switch') que permite acumular de uma forma aditiva os pulsos emitidos pelo 'pacemaker': hipoteticamente a uma frequência de $1 \mathrm{~Hz}$ teríamos 4 pulsos acumulados ao fim de 4 segundos $(1 \mathrm{x} 1+1 \mathrm{x} 1+1 \mathrm{x} 1+1 \mathrm{x} 1)$. A velocidade ou frequência de emissão de pulsos do 'pacemaker' ao aumentar tem um efeito multiplicativo no total de pulsos acumulado: hipoteticamente se a frequência aumentasse $0.5 \mathrm{~Hz}$ por segundo teríamos 7 pulsos acumulados ao fim de 4 segundos $(1 \times 1+1 \times 1.5+1 \times 2+1 \times 2.5)$. 
conduzindo a estimativas de duração mais longas. Tal deverá passar-se apenas na condição de baixa intensidade, porque na de elevada intensidade, espera-se que mesmo em durações mais curtas o efeito de activação se sobreponha ao da atenção. Em suma, esperamos que o efeito da valência só se verifique na condição de menor intensidade, e em durações mais curtas (i.e., inferiores à média da série temporal utilizada: $1000 \mathrm{~ms}$ ). Prevemos portanto uma interacção tripla: valência x activação x duração.

No presente estudo cingimo-nos a durações inferiores a 2 segundos (neste caso de 400 a $1600 \mathrm{~ms}$ ), não só para prevenir possíveis estratégias de contagem (ver Droit-Volet et al., 2004), mas também de modo a captar apenas, e de forma clara, o efeito ascendente da activação (prevenindo o potencial decréscimo em durações mais longas, ver; Fridja et al., 1992). É de referir ainda que a partir de 2-3s existem evidências de interferências descontínuas na percepção de duração, provavelmente associadas a processos mnési$\cos$ (e.g., Fortin \& Couture, 2002).

\section{Método}

\section{Participantes}

Participaram voluntariamente neste estudo um total de 108 estudantes com idades compreendidas entre os 16 e os 39 anos $(M=21.39$, D.P. $=4.90)$, na sua maioria do género feminino $(72.2 \%)$. Os participantes foram distribuídos pelas condições experimentais definidas pelo delineamento experimental, de forma aleatória.

\section{Delineamento experimental}

As características e manipulações dos estímulos definiram o plano factorial misto: 2 (valência de expressão facial do estímulo: positiva, negativa) x 2 (activação da expressão facial: baixa, elevada) x 2 (duração dos estímulos: curta, 400, 600, 800 e 1000, e longa, $1000,1200,1400$ e 1600), com apresentação de uma condição de controlo (expressão facial neutra). Os factores valência e activação foram manipulados inter-sujeitos (4 grupos), sendo a duração dos estímulos manipulada intra-sujeitos.

\section{Material}

Foram seleccionadas 35 faces do conjunto MacBrain Face Stimulus Set (Tottenham Borscheid, Ellertsen, Marcus, \& Nelson, 2002) ${ }^{16}$. Consisti-

16 Os estímulos seleccionados foram os seguintes: 03F_AN_C, 03F_AN_O, 03F_HA_C,
03F_HA_X, 03F_NE_C, 08F_AN_C, 08F_AN_O, 08F_HA_C, 08F_HA_X, 08F_NE_C, 
ram em quatro conjuntos de sete faces cada, correspondendo a (a) positivo de baixo activação (faces de alegria, expressão pouco intensa), (b) positivo de elevada activação (faces de alegria, expressão muito intensa), (c) negativo de baixa activação (faces de raiva, expressão pouco intensa), (d) negativo de elevada activação (faces de raiva, expressão muito intensa), assim como um conjunto de sete faces com expressão neutra.

\section{Procedimento}

A manipulação experimental foi realizada em laboratório e as tarefas executadas em computadores HP Vectra VL420DT (monitores a $100 \mathrm{~Hz}$ ). Os estímulos foram apresentados e as respostas registadas através do programa de geração de experiências E-Prime 1.1 (Psychology Software Tools, Sharpsburg, PA, EUA), com uma precisão de \pm 1.0 ms.

Os participantes realizaram uma tarefa de bissecção temporal com uma série de durações extremas de 400 e 1600, num rácio 1:4 (e.g., Allan \& Gibbon, 1991; Wearden, 1991), que consiste numa fase de treino e numa fase de teste. Durante a fase de treino inicial foram apresentados aos participantes estímulos neutros (quadrados pretos), 5 vezes alternadamente para as durações standard de referência designadas como curta $(400 \mathrm{~ms})$ e longa $(1600 \mathrm{~ms})$. Em cada uma das apresentações foram dadas instruções para que os participantes indicassem a duração do estímulo pressionando no teclado do computador as teclas amarela ('S') ou branca ('L') correspondentes (ou vice-versa, contrabalanceamento da lateralidade inter-sujeitos). Ainda na fase de treino os participantes realizaram seguidamente 8 ensaios com o mesmo estímulo, com as durações curta ou longa a sucederem com $50 \%$ de probabilidade. Nesta fase foi dado aos participantes um feedback da resposta depois de cada ensaio. Seguidamente os participantes executaram a fase de teste, em que foram mostradas as faces e solicitado que indicassem através do teclado se aquele estímulo fora apresentado durante um tempo próximo ao dos estímulos curtos ou longos aprendidos na fase de treino. Além das durações standard (400 e $1600 \mathrm{~ms}$ ) os estímulos foram apresentados com 5 durações intermédias $(600,800,1000,1200$ e $1400 \mathrm{~ms})$.

Os ensaios experimentais foram realizados em 7 blocos compostos por 14 estímulos cada, perfazendo um total de 98 ensaios por participante. Em cada bloco, cada tipo de estímulo (i.e., emocional e neutro) foi apresentado 7

10F_AN_C, 10F_AN_O, 10F_HA_C, 10F_HA X, 10F_NE_C, 21M AN C,

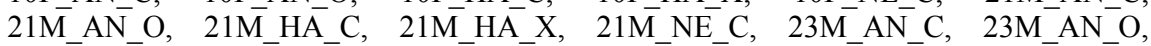
23M-HA_C, 23M_HA X, 23M-NE-C, 24M AN_C, 24M-AN-O, 24M-HA_C, 24M_HA_X, 24M_NE_C, 26M_AN_C, 26M_AN_O, 26M_HA_C, 26M_HA_X, 26M_NE_C. Foram escolhidas 15 faces femininas (3 actores) e 20 faces masculinas (4 actores), $\overline{d e}$ homens e mulheres adultos e caucasianos. 
vezes por cada duração referida, em sequência aleatória. As 7 imagens de cada tipo de estímulo foram contrabalançadas entre os blocos, de modo a que cada imagem fosse apresentada uma vez para cada duração. O tempo entre ensaios variou aleatoriamente entre 1000 e 2000 ms para evitar automatização da resposta.

\section{Resultados}

Cálculo dos índices de percepção temporal. Para a análise dos enviesamentos temporais procedeu-se a várias transformações da função psicofísica, resultante das estimativas temporais na tarefa de bissecção. Esta função sigmoidal constitui a variação da proporção de respostas longas para cada uma das durações dos estímulos (ver figura 1). Os enviesamentos traduzem-se em desvios para a esquerda (i.e., sobrestimativas) ou para a direita (i.e., subestimativas) das funções associadas às manipulações (i.e., activação e valência dos estímulos) relativamente à função psicofísica de referência (i.e., faces neutras) (e.g., Allan \& Gibbon, 1991; Droit-Volet et al., 2004; Wearden, 1991).

Utilizámos dois indicadores de enviesamentos associados à função psicofísica: os seus integrais e os seus pontos de bissecção. Os integrais da função psicofísica em termos matemáticos representam as áreas abaixo das curvas. Calculámos as diferenças dos integrais associados a cada uma das expressões faciais relativamente à das faces neutras de referência. Assim, diferenças positivas representam sobre-estimativas temporais, ou seja desvios à esquerda da função psicofísica, enquanto diferenças negativas representam sub-estimativas temporais (ver figura 2). A outra medida de enviesamento dos julgamentos de duração, associados a estímulos de diferente natureza emocional, é representada pelo 'cut point' (dos pontos de bissecção) decisional da distribuição das respostas aos estímulos emocionais e neutros. $\mathrm{O}$ ponto de bissecção traduz o ponto acima do qual o individuo decide dar uma resposta longa, e abaixo do qual decide dar uma resposta curta. Estes pontos foram calculados a partir dos parâmetros declive e intercepção às ordenadas e obtidos nas regressões lineares da zona de maior declive das funções psicofísicas individuais (entre 600 e 1400ms da série temporal). Quanto menor o ponto bissecção, maior a estimativa temporal (e.g., Allan, 2002; Church, \& Deluty, 1977; Droit-Volet et al., 2004). Assim, as diferenças negativas relativamente à referência representam sobre-estimativas temporais, enquanto as diferenças positivas representam sub-estimativas temporais. 
A

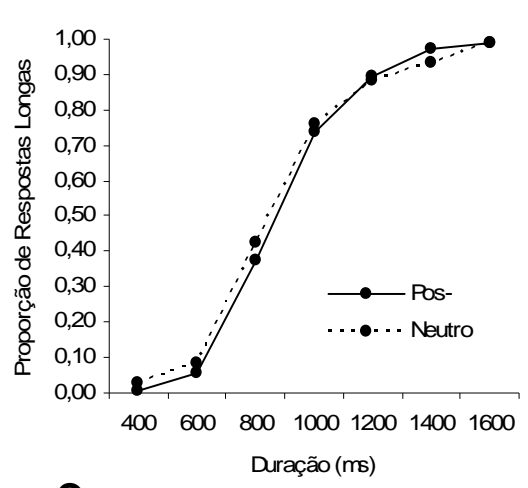

C

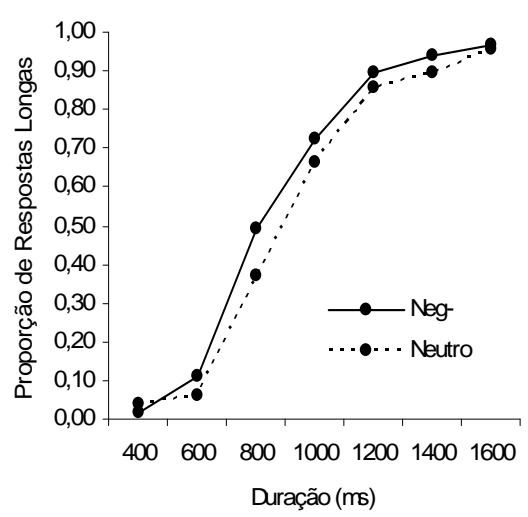

B

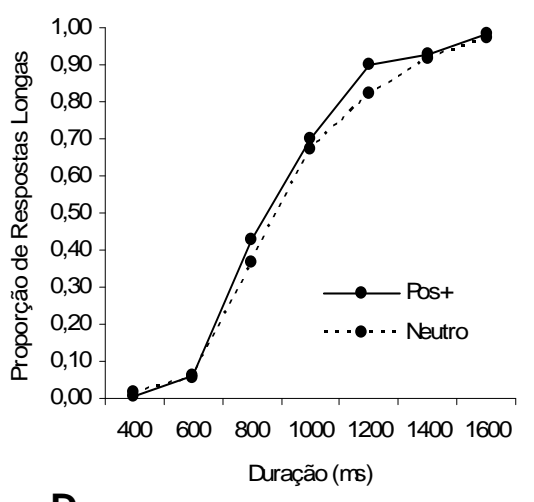

D

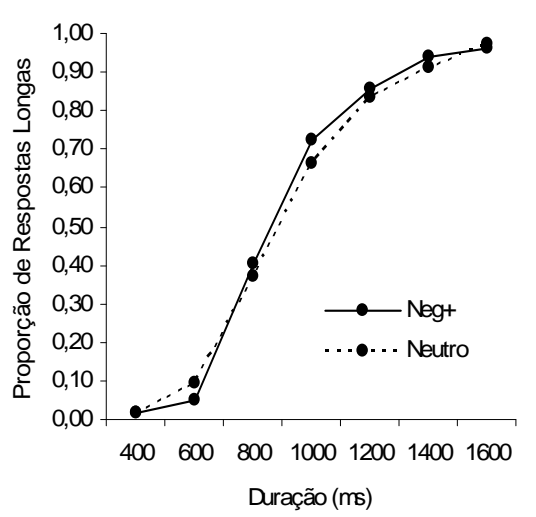

Figura 1. Proporção de respostas 'longas' em função das durações dos estímulos. Gráfico A: Funções psicofísicas para as expressões faciais neutras (referência) e positivas de baixa activação. Gráfico B: Funções psicofísicas para as expressões faciais neutras (referência) e positivas de elevada activação. Gráfico C: Funções psicofísicas para as expressões faciais neutras (referência) e negativas de baixa activação. Gráfico D: Funções psicofísicas para as expressões faciais neutras (referência) e negativas de elevada activação.

Efeitos da emoção (expressão facial) na percepção de tempo. Com vista a verificar o impacto geral das emoções na percepção temporal, foi realizada uma análise de variância (ANOVA) aos integrais das funções psicofísicas, com dois factores intra-participantes: emoção (estímulos emocio- 
nais vs neutros) ${ }^{17}$ e duração (curta, $<1000 \mathrm{~ms}$, vs longa, $\left.>1000 \mathrm{~ms}\right)^{18}$. Esta análise revelou apenas a presença do efeito esperado das emoções, $F(1$, 107) $=13.770, p<.001, \eta=.114$, a indicar uma sobre-estimativa temporal das faces emocionais $(M=.707, D . P .=.123)$, relativamente às faces neutras $(M$ $=.681, D . P .=135)$. A ausência da interacção entre emoção e duração, $F(1$, $107)=1.517, p=.221, \eta=.014$, indica que a sobre-estimativa temporal ocorreu uniformemente em toda a série temporal (para durações mais curtas e mais longas). Foi realizada uma ANOVA adicional com o mesmo plano factorial aos pontos de bissecção (p.b.) das curvas psicofísicas. Esta revelou, tal como para os integrais da função psicofísica, apenas a presença do efeito geral das emoções, $F(1,106)=10.706, p<.001, \eta=.091$ (p.b. dos estímulos emocionais: $M=892, D . P .=134$; p.b. dos estímulos neutros: $M=917, D . P .=156)$.

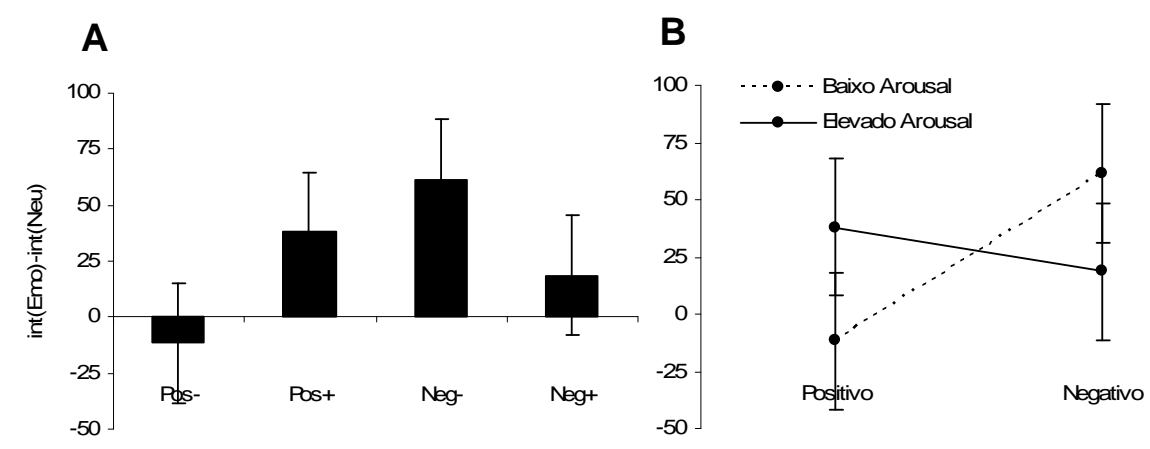

Figura 2. Gráfico A: Valores diferenciais dos integrais das funções psicofísicas das expressões faciais emocionais relativamente à referência (faces neutras, zero). Gráfico B: Representação ortogonal da valência e activação das expressões faciais para os integrais.

Efeitos das dimensões emocionais (valência e activação) na percepção de tempo. De modo a explorarmos os padrões de resposta dos indivíduos, realizámos uma ANOVA mista aos diferenciais dos integrais das funções psicofísicas das faces emocionais, relativamente às faces neutras, tendo

17 Nesta análise não foram distinguidos os tipos de emoção correspondentes à manipulação (definidos inter-participantes).

18 Estas durações (curta $<1000 \mathrm{~ms}$ e longa $>1000 \mathrm{~ms}$ ) foram escolhidas por corresponderem ao ponto médio da série temporal utilizada na tarefa de bissecção, em concordância com estudos anteriores (e.g., Droit-Volet et al., 2004). 
como factores inter-participantes a valência e a activação e como factores intra-participantes a duração. Os resultados sugerem que apenas a valência tem um efeito principal na percepção temporal $F(1,104)=3.944, p<.05$ $\eta=.037$ (estímulos positivos: $M=14.03, D . P .=71.32$; estímulos negativos: $M=40.11, D . P .=76.07)$. No entanto, tal como esperado, este efeito é moderado pela activação $F(1,104)=11.58, p<.001, \eta=.100$ (ver figura 2 ). A análise de contrastes post hoc revelou que, na condição de baixa activação os estímulos negativos $(M n e g-=61.54$, D.P. $=71.49)$ foram sobre-estimados relativamente aos positivos (Mpos- $=-11.64, D . P .=79.35), t(104)=3.778$, $p<.001$, enquanto na condição de elevada activação não existem diferenças entre os estímulos negativos $(M n e g+=18.68, D . P .=75.78)$ comparativamente aos positivos $(M$ pos $+=37.93$, D.P. $=54.03), t(104)=1.011, p=.314)$.

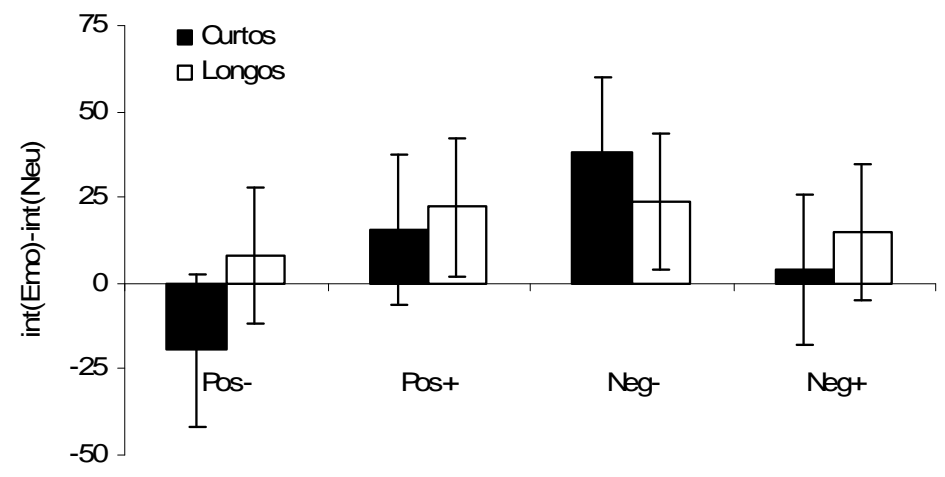

Figura 3. Valores diferenciais dos integrais das funções psicofísicas das expressões faciais emocionais relativamente à referência (faces neutras, zero), para as durações longas (>1000 ms) e para as durações curtas $(<1000 \mathrm{~ms})$.

Relevante para a compreensão deste efeito interactivo é o facto de este ser moderado pela duração. A interacção tripartida de duração $\mathrm{x}$ valência $\mathrm{x}$ activação, $F(1,104)=3.423, p=.067$, sugere que, apesar dos padrões de impacto das dimensões emocionais (valência e activação) na percepção de tempo serem aparentemente semelhantes nas estimativas para as durações curtas $(<1000 \mathrm{~ms})$ e longas $(>1000 \mathrm{~ms})$, (ver figura 3 onde a interacção apenas atinge significância estatística para as durações curtas $(t(104)=3.961$, $p=.0001)$, a análise de contrastes post hoc revelou que, na condição de baixa activação, os estímulos negativos (Mneg- $=37.91, D . P .=43.88)$ foram sobre-estimados relativamente aos positivos $(M$ pos- $=-19.58, D . P .=54.92)$, 
$t(104)=4.600, p=.00001$, enquanto na condição de elevada activação não existiram diferenças entre os estímulos negativos $(M$ neg $+=3.86, D . P .=$ 49.41) e os positivos $(M p o s+=15.76$, D.P. $=31.33), t(104)=.970, p=.334)$. Esta observação aponta para uma maior contribuição do padrão diferencial, para os efeitos gerais, das durações mais curtas da série temporal.

Estes dados são corroborados pela ANOVA, utilizando os pontos de bissecção. Esta análise detectou igualmente um efeito geral (embora marginal) da valência, $F(1,104)=3.875, p=.051, \eta=.036$ (estímulos positivos: $M=$ 9.33$, D.P. $=90.16$; estímulos negativos: $M=-41.70, D . P .=102.27)$, mas não da activação. A interacção entre valência e activação foi significativa, $F(1,104)=20.488, p<.0001, \eta=.165$. A análise de contrastes post hoc revelou que, em baixa activação, os estímulos negativos $(M$ neg- $=-83.10, D . P .=$ 91.42) foram sobre-estimados relativamente aos positivos (Mpos- $=27.93$, $D . P .=102.63), t(104)=3.032, p<.001$, enquanto em elevada activação os estímulos negativos $(M n e g+=-.30, D . P .=97.04)$ foram sub-estimados, comparativamente aos positivos $\left(M\right.$ pos $\left.^{+}=-44.03, D . P .=59.99\right), t(104)=$ $3.364, p=.001$.

\section{Discussão}

Os dados obtidos neste estudo, tal como esperado, sugerem que as faces emocionais, em comparação com as faces neutras, foram percebidas como tendo durações mais longas. Isto foi demonstrado de várias formas. Por um lado, as faces emocionais aumentaram os integrais das funções psicofísicas, e por outro, diminuíram os pontos de bissecção, indicando um enviesamento para dar respostas longas. Estes dados estão em consonância com os diversos estudos que observaram o mesmo efeito de sobre-estimativa temporal das faces emocionais (ver Droit-Volet et al. 2004; Effron et al., 2006; Fernandes et al., 2006; Fernandes \& Garcia-Marques 2008b; Gil et al., 2007, Mondillon et al., 2007; Thayer \& Schiff; 1975; Tipples, 2008). Esta tendência geral para sobre-estimar a duração dos estímulos emocionais parece reflectir mais um efeito de activação, mais elevado nos estímulos neutros, do que um efeito atencional. De acordo com os modelos descritos, um aumento de activação actua no relógio interno promovendo uma aceleração do 'pacemaker', emitindo consequentemente mais pulsos por unidade de tempo, conduzindo, por sua vez, a uma sobre-estimativa temporal.

Adicionalmente, os estímulos negativos foram julgados como tendo durações mais longas do que os estímulos positivos. Tal impacto da valência sugere a possibilidade de as faces negativas (raiva) acelerarem o relógio interno mais do que as faces positivas (alegria), o que é habitualmente atribuído apenas à activação. De facto existem evidências que as emoções nega- 
tivas produzem respostas neurais, fisiológicas, cognitivas e comportamentais mais pronunciadas do que as produzidas pelos afectos neutros ou positivos (e.g., Cacioppo \& Gardner, 1999; Ito, Larsen, Smith, \& Cacioppo, 1998; Larsen, Norris, \& Cacioppo, 2003). Particularmente, este viés da negatividade tem sido observado ao nível da excitabilidade (i.e., activação). Embora a raiva e a alegria sejam ambas consideradas emoções tendencialmente com elevada activação, alguns estudos sugerem níveis de activação ligeiramente superiores para a raiva (e.g., Russell, 1983). Tal facto poderia sugerir que o efeito de valência observado traduziria apenas um impacto da activação, uma vez que as faces de maior activação (raiva) foram sobre-estimadas em relação às de menor activação (alegria). Estes dados são consistentes com estudos anteriores (Effron et al., 2006; Droit-Volet et al., 2004; Thayer \& Schiff, 1975; Tipples, 2008), que mostraram igualmente estimativas temporais maiores para as faces de raiva do que para as de alegria. Contudo, e incongruentemente com esta interpretação, os nossos dados não revelaram um efeito de sobre-estimativa da intensidade das expressões faciais. Observa-se, no entanto, uma moderação dos efeitos da valência pelo nível de activação associado aos estímulos. Quando a activação foi baixa, as diferenças de valência foram na direcção de sub-estimativa de estímulos positivos relativamente aos negativos, e quando a activação foi elevada, verificámos uma sub-estimativa dos estímulos negativos. Apesar de esta interacção ser inversa às previsões, sugere uma interferência de processos atencionais concorrentes com os efeitos da activação na aceleração do relógio interno. É, portanto, indicativo de que estas duas variáveis associadas ao processamento dos estímulos emocionais não são independentes (nos efeitos que produzem nas estimativas temporais desses estímulos). Esperar-se-ia que as faces positivas fossem sobre-estimadas em relação às negativas na condição de baixa intensidade, ou seja, que os efeitos de desvio da atenção promovidos pelas faces negativas (e.g., Fox, et al., 2000; Öhman et al., 2000; Bonifacci et al., 2008) se sobrepusessem aos da activação apenas quando esta fosse menos elevada. Este resultado pode ser sustentado de várias formas. Primeiro, estudos revelam que, quando os estímulos ameaçadores (e.g., expressão de raiva) são irrelevantes para a tarefa (neste caso, de estimação temporal), podem tornar-se distractores, resultando em efeitos de interferência, sendo que estes efeitos resultam em função da distintividade (relevância), competindo pela atenção selectiva implicada (Compton, 2003). Ora, a apreensão da relevância do estímulo ("ameaçador") é maior quando a expressão de raiva é mais intensa. Segundo, e neste sentido, o facto da interacção entre valência e activação só ser significativa para durações inferiores a $1000 \mathrm{~ms}$ (as durações mais curtas da série temporal), permite-nos inferir uma maior competição entre os efeitos atencionais e os de activação, comparativamente a durações mais longas (e.g., Burle \& Casini 2001; Droit-Volet et al., 2004; Fortin \& Couture, 
2002). Ou seja, os efeitos de desvio da atenção promovidos pelas faces de raiva só se sobrepõem aos da activação quando a intensidade da expressão é maior e quando a duração dos estímulos é mais curta.

Comparativamente aos estudos de Angrilli e colaboradores (1997) e de Noulhiane e colaboradores (2007), que testaram igualmente a interacção entre valência e activação, o padrão de dados observado é totalmente distinto de ambos os estudos, e de estes entre si. Contudo, na origem destas aparentes incongruências podem estar diferenças nas manipulações. Em primeiro lugar, porque utilizam modalidades sensoriais distintas. Um conjunto de estudos tem mostrado que os estímulos sonoros são julgados como tendo durações mais longas do que os estímulos visuais (e.g., Droit-Volet, Meck, \& Penney, 2007; Penney, Gibbon, \& Meck, 2000; Wearden, Edwards, Fakhri, \& Percival, 1998). Wearden et al. (1998) sugerem a existência de uma menor variabilidade no efeito da atenção ${ }^{19}$ e uma maior aceleração do relógio interno para os estímulos sonoros. Em segundo lugar, as propriedades sensoriais dos estímulos sonoros permitem a extracção de diferentes pistas temporais relativamente aos estímulos visuais (ver van Wassenhove, et al., 2008): os primeiros são dinâmicos, variando ao longo do tempo, enquanto os segundos são estáticos. Por exemplo, um estímulo sonoro de um acidente automóvel (tipo de estímulos utilizado por Noulhiane et al., 2007) terá o seu culminar no final do estímulo, promovendo um pico de activação apenas no final desse evento. No nosso caso, ao utilizarmos faces, diminuímos a variabilidade, quer da frequência espacial, quer da complexidade dos estímulos, que se sabe interferirem na percepção de tempo (e.g., Block, 1992; Fortin, Rousseau, Bourque, \& Kirouac, 1993; Penton-voak et al., 1996). Em terceiro lugar, o protocolo utilizado nos estudos de Angrilli e colaboradores (1997) e de Noulhiane e colaboradores (2007) acrescenta ruído na própria estimativa de tempo uma vez que, em cada ensaio, é pedido aos participantes que avaliem a intensidade e a valência do estímulo. Ou seja, introduzem uma tarefa concorrente que, como é sabido, reduz os recursos atencionais para processar a informação temporal (e.g., Brown, 1997; Zakay \& Yehoshua, 1989). De facto Angrilli et al. (1997) obtiveram, em termos gerais, sub-estimativas da duração dos estímulos. Em quarto lugar, outro aspecto a ter em conta é a janela temporal utilizada, superior a $2 \mathrm{~s}$ no caso de Angrilli et al. (1997) e de

19 Tem sido sugerido que a menor variabilidade das estimativas temporais de estímulos sonoros relativamente aos visuais se deve à estabilidade da abertura do interruptor ('switch') que marca o inicio da contagem e a acumulação de pulsos emitidos pelo 'pacemaker'. Ao longo do evento, este interruptor pode abrir e fechar várias vezes, dependendo da flutuação da atenção entre a informação temporal e não-temporal do estímulo. A latência da abertura deste interruptor parece ser mais estável, também de estímulo para estímulo, para a modalidade auditiva do que para a visual (ver Wearden et al., 1998; van Wassenhove et al., 2008). 
Noulhiane et al. (2007) e inferior a 2 s no presente estudo, o que pode ter implicações importantes, dada a própria dinâmica da percepção dos estímulos emocionais. Em quinto lugar, a tarefa de bissecção utilizada no presente estudo, ao utilizar uma série com vários pontos temporais (neste caso sete: $400,600,800,1000,1200,1400,1600 \mathrm{~ms}$ ), permite diminuir a variabilidade associada a estimativas referentes apenas a pontos absolutos (i.e., durações específicas: por exemplo, 2, 4 ou 6 s), utilizadas nos estudos de Angrilli et al. (1997) e de Noulhiane et al. (2007); refira-se que os efeitos referidos por estes dois estudos são moderados pela duração.

Em termos gerais, os dados do presente estudo revelam que a percepção temporal de estímulos emocionais é sensível aos vários componentes emocionais desses estímulos. Deste modo, é relevante em estudos futuros testar a relação entre o impacto afectivo que estes estímulos têm efectivamente no indivíduo, na percepção da duração desses estímulos (controlando os níveis de activação e os recursos atencionais dispendidos). Sugere também que é sensível à dinâmica temporal das emoções, uma vez que os efeitos variam em função da própria duração dos estímulos - aspecto particular ainda pouco explorado no amplo campo de investigação das emoções. Tendo em conta os resultados discrepantes em relação a estudos anteriores, que manipulam ortogonalmente a valência e a activação, é aconselhável que em futuros estudos se atenda aos vários aspectos referidos que potencialmente interferem na própria estimação temporal.

Certas limitações deste estudo necessitam ser sublinhadas. Primeiro, a generalização dos efeitos da valência aqui referidos torna-se restrita, se optarmos por uma abordagem categorial das emoções e suas funções adaptativas. Por exemplo, enquanto a raiva em termos motivacionais promove a preparação para a acção, aumentando os níveis de activação (Izard \& Ackerman, 2000), a vergonha ou a culpa, que também podem estar associadas a níveis de elevada activação, são emoções morais e sociais que captam a atenção através da auto-consciência reflexiva (Lewis, 1993): esperar-se-ia que a raiva levasse a uma sobre-estimativa, enquanto a vergonha e a culpa levassem a uma sub-estimativa temporal. Por outro lado, as várias emoções podem suscitar níveis variados de activação, num crescendo tristeza $<$ medo $<$ raiva (e.g., Russell, 1983), mesmo sendo emoções da mesma valência, conduzindo a estimativas temporais crescentes (ver Droit-Volet et al., 2004).

Em segundo lugar, apesar de existir concordância com os resultados de estudos que manipulam alegria e raiva (Droit-Volet et al., 2004; Effron et al., 2006, Thayer \& Schiff, 1975; Tipples, 2008), ao contrário das nossas observações, alguns estudos verificaram sobre-estimativas temporais dos estímulos positivos comparativamente à dos estímulos negativos (Edmonds, 1981; Hawkings et al., 1988; Xuan \& Xiaolin, 2007). Quer num sentido, quer noutro, de um ponto de vista estrito, estes estudos não podem ser com- 
parados, uma vez que não sabemos qual o nível de activação associado aos estímulos utilizados em cada uma das manipulações.

Em terceiro lugar, a reactividade individual aos estímulos emocionais pode variar, aspecto que não foi controlado, e é relevante, uma vez que as manipulações foram feitas inter-participantes. Existem evidências na literatura acerca da variabilidade inter-individual das emoções na percepção do tempo (Tipples, 2007). Outro aspecto particular a ter em conta é a reactividade às expressões de raiva, que pode variar do evitamento (resposta aversiva) à agressão (resposta aproximativa) (ver Compton, 2003), principalmente em indivíduos com maior ansiedade, ou em condições que promovam a ansiedade (Fox, Russo,\& Dutton, 2002; Schutter, de Haan, \& van Honk, 2004): no caso de uma resposta aversiva, ao deslocar a atenção do estímulo o indivíduo poderá não atender igualmente à informação temporal.

Em quarto lugar, poder-se-á estar perante outros mecanismos de estimativa temporal para além dos implicados directamente na informação temporal, partindo do axioma genericamente aceite de que a informação temporal implica a existência de um relógio interno (e.g., Block, 1990; Gibbon \& Church, 1984; Gibbon, Church, \& Meck, 1984; Treisman, 1963). Existem evidências na literatura referindo que, a par destes mecanismos, podem co-ocorrer outros processos, por exemplo, associados à fluência de processamento (e.g., Witherspoon \& Allan 1985). Os estímulos processados mais fluentemente são sobre-estimados relativamente aos menos fluentes (e.g., palavras vs não-palavras, Reber, Zimmermann \& Wurtz, 2004). Se assumirmos que as expressões faciais mais intensas são mais distintas das menos intensas, poderão ser processadas mais fluentemente, e por uma falsa atribuição à duração (e.g., Whittlesea, 1993), serem sobre-estimadas.

Em quinto lugar, o facto de as manipulações das condições emocionais serem realizadas inter-participantes não nos permite controlar, para efeitos de contraste ou de assimilação relativamente ao contexto, neste caso as expressões faciais neutras (e.g., Cupchik \& Poulos, 1984; Schwarz \& Bless, 1992). A duração dos estímulos é determinada igualmente pela comparação entre tipos diferentes de estímulos: a questão que se coloca é se determinadas emoções ou diferentes níveis de activação promovem sempre efeitos de contraste (no sentido da sobre-estimativa relativamente aos estímulos neutros) ou, por vezes, efeitos de assimilação (i.e., sub-estimativa).

Em sexto lugar, as interpretações aqui descritas ficam confinadas à janela temporal estudada. Como referido anteriormente, a dinâmica temporal das emoções, por implicar variações atencionais e nos níveis de activação ao longo do tempo, requer novos estudos que confirmem inequivocamente a co-ocorrência dinâmica destes componentes emocionais com os enviesamentos temporais, no sentido das previsões dos modelos baseados num relógio 
interno. Para tal, é necessário igualmente estudar o processo em diferentes janelas temporais, ou com diferentes latências de resposta (por exemplo).

Em suma, aparentemente estes resultados reflectem a possibilidade de que a percepção de duração de estímulos emocionais (neste caso de expressões faciais) seja sensível aos vários componentes emocionais envolvidos no processamento desses mesmos estímulos (valência e activação). Embora estes dados permitam enunciar a hipótese de que factores concorrenciais, atencionais e de activação, induzidos no indivíduo, estejam implicados na interferência nos mecanismos de processamento temporal, são até ao momento inconclusivos e apelam a que um maior número de investigações sobre o tema seja realizado.

\section{Referências}

Allan, L. G. (2002). The location and interpretation of the bisection point. The Quarterly Journal of Experimental Psychology, 55B, 43-60.

Allan, L., \& Gibbon, J. (1991). Human bisection at the geometric mean. Learning and Motivation, 22, 39-58.

Almeida, I., van Asselen, M., \& Castelo-Branco, M. (2010). Separable effects of stimulus duration and awareness on skin conductance responses to emotional facial expressions. Livro de Resumos do $5^{\circ}$ Encontro Nacional de Investigação da Associação Portuguesa de Psicologia, Braga, Portugal, 26 e 27 de Março de 2010.

Angrilli, A., Cherubini, P., Pavese, A., \& Manfredini, S. (1997). The influence of affective factors on time perception. Perception \& Psychophysics, 59, 972-982.

Atkinson, A. P., \& Adolphs, R. (2005). Visual emotion perception: Mechanisms and processes. In L. F. Barrett, P. M. Niedenthal, \& P. Winkielman (Eds.), Emotion and consciousness (pp. 150-182). New York: Guilford Press.

Block, R. A. (1990). Models of psychological time. In R. A. Block (Ed.), Cognitive models of psychological time (pp. 1-35). Hillsdale, NJ: Erlbaum.

Block, R. A., \& Zakay, D. (1997). Prospective and retrospective duration judgments: A meta-analytic review. Psychonomic Bulletin \& Review, 4, 184-197.

Block, R. A. (1992). Prospective and retrospective duration judgment: The role of information processing and memory. In F. Macar, V. Pouthas, \& W. I. Friedman (Eds.), Time, action and cognition: Towards bridging the gap (pp. 141-152), Dordrecht: Kluwer Acad.

Bonifacci, P., Ricciardelli, P., Lugli, L., \& Pellicano, A. (2008). Emotional attention: Effects of emotion and gaze direction on overt orienting of visual attention. Cognitive Processing, 9, 127-135.

Bradley, M. M. (2000). Emotion and motivation. In J. T. Cacioppo, L. G. Tassinary, \& G. Berntson (Eds.), Handbook of psychophysiology. (pp. 602-642). New York: Cambridge University Press. 
Bradley, M. M., \& Lang, P. J. (1999). International affective digitized sounds (IADS): Stimuli, instruction manual and affective ratings (Tech. Rep. No. B-2). Gainesville, FL: University of Florida, Center for Research in Psychophysiology.

Brown, S. W. (1997). Attentional resources in timing: Interference effects in concurrent temporal and nontemporal working memory tasks. Perception \& Psychophysics, 59, 1118-1140.

Buhusi, C. V., \& Meck, W. H. (2005). Interval timing with gaps and distracters: Evaluation of the ambiguity, switch, and time-sharing hypotheses. Journal of Experimental Psychology: Animal Behavior Processes, 3, 329-338.

Buhusi, C. V., \& Meck, W. H. (2005). What makes us tick? Functional and neural mechanisms of interval timing. Nature Reviews Neuroscience, 6, 755-765.

Buhusi, C. V. (2003). Dopaminergic mechanisms of interval timing and attention. In W. H. Meck (Ed.), Functional and neural mechanisms of interval timing (pp. 317-338). Boca Raton, FL: CRC Press.

Burle, B., \& Casini, L. (2001). Dissociation between activation and attention effects in time estimation: Implication for internal clock models. Journal of Experimental Psychology: Human Perception and Performance, 27, 195-205.

Cacioppo, J. T., \& Gardner, W. L. (1999). Emotion. Annual Review of Psychology, $50,191-214$

Campbell, L. A., \& Bryant, R. A. (2007). How time flies: A study of novice skydivers. Behaviour Research and Therapy, 45, 1389-1392.

Casini, L., \& Macar, F. (1997). Effects of attention manipulation on judgments of duration and of intensity in the visual modality. Memory \& Cognition, 25, 812-818 .

Chebat, J.-C., \& Gelinas-Chebat, C. (1995). The impact of mood on time perception, memorization, and acceptance of waiting. Genetic, Social \& General Psychology Monographs, 121, 411-425

Cheng, R.-K., MacDonald, C. J., \& Meck, W. H. (2006). Differential effects of cocaine and ketamine on time estimation: Implications for neurobiological models of interval timing. Pharmacology, Biochemistry and Behavior, 85, 114-122.

Church, R. M., \& Deluty, M. Z. (1977). Bisection of temporal intervals. Journal of Experimental Psychology: Animal Behavior Processes, 3, 216-228.

Compton, R. J., (2003). The interface between emotion and attention: A review of evidence from psychology and neuroscience. Behavioral and Cognitive Neuroscience Reviews, 2, 115-129.

Cupchik, G. C., \& Poulos, C. X. (1984). Judgments of emotional intensity in self and others: The effects of stimulus context, sex, and expressivity. Journal of Personality and Social Psychology, 46, 431-439.

Curton, E. D., \& Lordahl, D. S. (1974). Effects of attentional focus and arousal on time estimation. Journal of Experimental Psychology, 103, 861-867.

Cuthbert, B. N., Bradley, M. M., \& Lang, P. J. (1996). Probing picture perception: Activation and emotion. Psychophysiology, 33, 103-111.

Delay, E. R., \& Mathey, M. E. (1985). Effects of ambient noise on time estimation by humans. Perceptual and Motor Skills, 61, 415-419. 
Droit-Volet, S., \& Meck, W. H. (2007). How emotions colour our perception of time. Trends in Cognitive Sciences, 11, 504-513.

Droit-Volet, S., \& Wearden, J. (2002). Speeding up an internal clock in children? Effects of visual flicker on subjective duration. The Quaterly Journal of Experimental Psychology, 55B(3), 193-211.

Droit-Volet, S., Brunot, S., \& Niedenthal, P. (2004). Perception of the duration of emotional events. Cognition \& Emotion, 18, 849-858.

Droit-Volet, S., Meck, W. H., \& Penney, T. B. (2007). Sensory modality and time perception in children and adults. Behavioural Processes, 74, 244-250.

Edmonds, E., Cahoon, D., \& Bridges, B. (1981). The estimation of time as a function of positive, neutral or negative expectancies. Bulletin of the Psychonomic Society, 17, 259-260.

Effron, D. A., Niedenthal, P. M., Gil, S., \& Droit-Volet, S. (2006). Embodied temporal perception of emotion. Emotion, 6, 1-9.

Ekman, P. (1992). An argument for basic emotions. Cognition and Emotion, 6, 169 -200 .

Fernandes, A. C., \& Garcia-Marques, T. (2008a). Affective interference in temporal perception. Abstract of XXIX International Congress of Psychology, Berlin, Germany, July 20-25.

Fernandes, A. C., \& Garcia-Marques, T. (2008b). Timing task and affective arousal modulation of time perception. Livro de Resumos do $3^{\circ}$ Encontro Nacional da Associação Portuguesa de Psicologia Experimental, Faro, Portugal, 28 e 29 de Março.

Fernandes, A. C., Garcia-Marques, T., \& Sá, L. (2006). Interferência afectiva na percepção temporal. Livro de Resumos do VI Simpósio Nacional de Investigação em Psicologia, Associação Portuguesa de Psicologia, Évora, Portugal, 29 e 30 de Novembro.

Fortin, C., \& Couture, E. (2002). Short-term memory and time estimation: Beyond the 2-second "critical value". Canadian Journal of Experimental Psychology, 56, 120-127.

Fortin, C., Rousseau, R., Bourque, P., \& Kirouac, E. (1993). Time estimation and concurrent nontemporal processing: Specific interference from short-term-memory demands. Perception \& Psychophysics, 53, 536-548.

Fox, E., Lester, V., Russo, R., Bowles, R. J., Pichler, A., \& Dutton, K. (2000). Facial expressions of emotion: Are angry faces detected more efficiently?. Cognition \& Emotion, 14, 61-92.

Fox, E., Russo, R., \& Dutton, K. (2002). Attentional bias for threat: Evidence for delayed disengagement from emotional faces. Cognition and Emotion, 16, $355-$ -379 .

Fox, R., Bradbury, P. A., Hampton, I. F., \& Legg, C. F. (1967). Time judgment and body temperature. Journal of Experimental Psychology, 75, 88-96.

Fraisse P. (1957) Psychologie du temps. Paris: PUF.

Fraisse, P. (1984). Perception and estimation of time. Annual Review of Psychology, 35, 1-36.

Frijda, N. H. (1986). The emotions. Cambridge, UK: Cambridge University Press. 
Fridja, N. H., Ortony, A., Sonnemans, J., \& Clore, G. L. (1992). The complexity of intensity: Issues concerning the structure of emotion intensity. In M. Clark (Ed.), Review of Personality and Social Psychology, 13, 60-89.

Friedman, W. J. (1990). About time. Inventing the fourth dimension. Cambridge, MA: MIT.

Gibbon, J., \& Church, R. (1984). Sources of variance in an information processing theory of timing. In H. Roitblat, T. Bever \& H. Terrace (Eds.), Animal cognition (pp. 465-488). London: Erlbaum.

Gibbon, J., Church, R., \& Meck, W. (1984). Scalar timing in memory. Annals of the New York Academy of Sciences, 423, 52-77.

Gil, S., Niedenthal, P., \& Droit-Volet, S. (2007). Anger and temporal perception in children. Emotion, 7, 219-225.

Gupta, S., \& Cummings, L. (1986). Perceived speed of time and task affect. Perceptual and Motor Skills, 63, 971-980.

Hawkins, W., French, L., Crawford, B., \& Enzle, M. (1988). Depressed affect and time perception. Journal of Abnormal Psychology, 97, 275-280.

Ito, T. A., Larsen, J. T., Smith, N. K., \& Cacioppo, J. T. (1998). Negative information weighs more heavily on the brain: The negativity bias in evaluative categorizations. Journal of Personality and Social Psychology, 75, 887-900.

Izard, C. E., \& Ackerman, B. P. (2000). Motivational, organisational and regulatory functions of discrete emotions. In M. Lewis \& J. M. Haviland-Jones (Eds.), Handbook of emotions (2nd ed.) (pp. 253-265). New York: Guilford Press.

James, W. (1890). The principles of psychology. Dover Publications.

Killeen, P. R., \& Fetterman, J. G. (1988). A behavioral theory of timing. Psychological Reviews, 95, 274-295.

Lang P. J., Bradley M. M., \& Cuthbert B. N. (1995). International Affective Picture System (IAPS): Technical manual and affective ratings (Tech. Rep. No. A-4). Gainesville: University of Florida, Center for Research in Psychophysiology.

Lang, P. J., Bradley, M. M., \& Cuthbert, B. N. (1990). Emotion, attention, and the startle reflex. Psychological Review, 97, 377-395.

Langer, J., Wapner, S., \& Werner, H. (1961). The effect of danger upon the experience of time. American Journal of Psychology, 74, 94-97.

Larsen, J. T., Norris, C. J., \& Cacioppo, J. T. (2003). Effects of positive and negative affect on electromyographic activity over zygomaticus major and corrugator supercilii. Psychophysiology, 40, 776-785.

Levenson, R.W., Ekman, P., \& Friesen, W.V. (1990). Voluntary facial action generates emotion-specific autonomic nervous system activity. Psychophysiology, $27,363-384$.

Lewis, M. (1993). Self-conscious emotions: Embarrassment, pride, shame and guilt. In M. Lewis, \& J. M. Haviland (Eds.), Handbook of emotions (pp. 563-594). New York: Guilford.

Macar, F., Grondin, S., \& Casini, L. (1994). Controlled attention sharing influences time estimation. Memory and Cognition, 22, 673-686. 
Maricq, A. V., Roberts, S., \& Church, R. M. (1981). Methamphetamine and time estimation. Journal of Experimental Psychology: Animal Behavior Processes, 7, 18-30.

Mondillon, L., Niedenthal, P. M., Gil, S., \& Droit-Volet, S. (2007). Imitation of in-group versus out-group members' facial expressions of anger: A test with a time perception task. Social Neuroscience, 2, 223-237.

Niedenthal, P. M. (2007). Embodying emotion. Science, 316, 1002-1005.

Noulhiane, M., Mella, N., Samson, S., Ragot, R., \& Pouthas, V. (2007). How emotional auditory stimuli modulate time perception. Emotion, 7, 697-704.

Noyes, R., \& Kletti, R. (1977). Depersonalization in response to life-threatening danger. Comprehensive Psychiatry, 18, 375-384.

Öhman, A., \& Soares, J. J. F. (1994). "Unconscious anxiety": Phobic responses to masked stimuli. Journal of Abnormal Psychology, 103, 231-240.

Öhman, A., Lundqvist, D., \& Esteves, F. (2001). The face in the crowd revisited: An anger superiority effect with schematic stimuli. Journal of Personality and Social Psychology, 80, 853-864.

Orme, J. (1969). Time experience and behaviour. Amsterdam: Elsevier.

Penney, T. B., Gibbon, J., \& Meck, W. H. (2000). Differential effects of auditory and visual signals on clock speed and temporal memory. Journal of Experimental Psychology: Human Perception and Performance, 26, 1770-1787.

Penton-voak, I. S., Edwards, H., Percival, A., \& Wearden, J. H. (1996). Speeding up an internal clock in humans? Effects of click trains on subjective duration. Journal of Experimental Psychology: Animal Behavior Processes, 22, 307-320.

Plutchik, R. (1962). The emotions: Facts theories and a new model. New York: Random House.

Pöppel, E. (1978). Time perception. In R. Held, H. W. Leibowitz \& H.-L. Teuber (Eds.), Handbook of sensory physiology: Vol. 8 (pp. 713-729). Berlin Heidelberg New York: Springer.

Reber, R., Zimmermann, T. D., \& Wurtz, P. (2004). Judgments of duration, figure-ground contrast and size for words and nonwords. Perception and Psychophysics, 66, 1105-1114.

Russell, J. A. (1980). A circumplex model of affect. Journal of Personality and Social Psychology, 39, 1161-1178.

Russell, J. A. (1983). Pancultural aspects of the human conceptual organization of emotions. Journal of Personality and Social Psychology, 39, 1281-1288.

Sabatinelli, D., Bradley, M. M., Fitzsimmons, J. R., \& Lang, P. J. (2005). Parallel amygdala and inferotemporal activation reflect emotional intensity and fear relevance. NeuroImage, 24, 1265-1270.

Schiff, W., \& Thayer, S. (1968). Cognitive and affective factors in temporal experience: Anticipated or experienced pleasant and unpleasant sensory events. Perceptual and Motor Skills, 26, 799-808.

Schiff, W., \& Thayer, S. (1970). Cognitive and affective factors in temporal experience: judgments of intrinsically and extrinsically motivated successful and unsuccessful performances. Perceptual and Motor Skills, 30, 895-902. 
Schutter, D. J., Hofman, D., \& van Honk, J. (2008). Fearful faces selectively increase corticospinal motor tract excitability: A transcranial magnetic stimulation study. Psychophysiology, 45, 345-348.

Schwarz, N., \& Bless, H. (1992). Constructing reality and its alternatives: An inclusion/exclusion model of assimilation and contrast effects in social judgment. In L. L. Martin \& A. Tesser (Eds.), The construction of social judgments (pp. 217-245). Hillsdale, NJ: Erlbaum.

Sokolov, Y. N. (1963). Perception and the conditioned reflex. New York: McMillan.

Staddon, J. E. R., \& Higa, J. J. (1999). Time and memory: Towards a pacemaker-free theory of interval timing. Journal of the Experimental Analysis of Behavior, 71, 215-251.

Stemmler, G., Aue, T., \& Wacker, J. (2007). Anger and fear: Separable effects of emotion and motivational direction on somatovisceral responses. International Journal of Psychophysiology, 66, 141-153.

Stetson, C., Fiesta, M. P., \& Eagleman, D. M. (2007). Does time really show during a frightening event?. PloS ONE, 2, e1295.

Sturt, M. (1925). The psychology of time. New York: Harcourt, Brace, \& World.

Tanaka, J. W., \& Farah, M. J. (2003). The holistic representation of faces. Advances in visual cognition. In M. A. Peterson \& G. Rhodes (Eds.), Perception of faces, objects, and scenes: Analytic and holistic processes. (pp. 53-71). New York, NY, US: Oxford University Press.

Thayer, S., \& Shiff, W. (1975). Eye-contact, facial expression, and the experience of time. The Journal of Social Psychology, 95, 117-124.

Tipples, J. (2008). Negative emotionality influences the effects of emotion on time perception. Emotion, 8, 127-131.

Tottenham, N., Borscheid, A., Ellertsen, K., Marcus, D. J., \& Nelson, C. A. (2002). Categorization of facial expressions in children and adults: Establishing a larger stimulus set. Journal of Cognitive Neuroscience, 14(Suppl.), S74.

Treisman, M. (1963). Temporal discrimination and the indifference interval: Implications for a model of the "Internal Clock". Psychological Monographies, 77, 13.

Treisman, M., Cook, N., Naish, P., \& McCrone, J. (1994). The internal clock: Electroencephalographic evidence for oscillatory processes underlying time perception. Quarterly Journal of Experimental Psychology, 47A, 241-289.

Ursano, R., Fullerton, C., Epstein, R., Crowley, B., Vance, K., \& Kao, T.-C. (1999). Peritraumatic dissociation and posttraumatic stress disorder following motor vehicle accidents. The American Journal of Psychiatry, 156, 1808-1810.

van Wassenhove, V., Buonomano, D. V., Shimojo, S., Shams, L. (2008). Distortions of subjective time perception within and across senses. PLoS ONE, 3, e1437.

Vercruyssen, M., Hancock, P. A., \& Mihaly, T. (1989). Time estimation performance before, during, and following physical activity. Journal of Human Ergology, 18(2), 169-179.

Watts, F. N., \& Sharrock, R. (1984). Fear and time estimation. Perceptual and Motor Skills, 59, 597-598. 
Wearden, J. H. (1991). Do humans possess an internal clock with scalar timing properties?. Learning and Motivation, 22, 59-83.

Wearden, J. H., \& Penton-Voak, I. S. (1995). Feeling the heat: Body temperature and the rate of subjective time, revisited. Quarterly Journal of Experimental Psychology, 48B, 129-141.

Wearden, J. H., Edwards, H., Fakhri, M., \& Percival, A. (1998). Why "sounds are judged longer than lights": Application of a model of the internal clock in humans. The Quarterly Journal of Experimental Psychology: Comparative and Physiological Psychology, 51, 97-120.

Whittlesea, B. W. A. (1993). Illusions of familiarity. Journal of Experimental Psychology: Learning, Memory, \& Cognition, 19, 1235-1253.

Winkielman, P., \& Cacioppo, J. T. (2001). Mind at ease puts a smile on the face: Psychophysiological evidence that processing facilitation increases positive affect. Journal of Personality and Social Psychology, 81, 989-1000.

Witherspoon, D., \& Allan, L. G. (1985). The effect of a prior presentation on temporal judgments in a perceptual identification task. Memory \& Cognition, 13, 101-111.

Xuan, Z., \& Xiaolin, Z., (2007). Time perception of emotional events. Progress in Natural Science, 17(13), 150-153.

Zakay, D., \& Yehoshua, T. (1989). Awareness of attention allocation and time estimation accuracy. Bulletin of Psychonomic Society, 27, 209-210. 